issues including governance and ownership, for which interdepartmental communication was efficient within the UHS. Specifically, the tracking system expanded the healthcare informatics system that pharmacists were familiar with, and its user-friendly interfaces for PPE providers and consumers helped expedite distribution processes. $^{5}$ The UHS and the TCDC have also promoted the system to increase the distribution channels, within which government offices may also allot masks to lessen the burden on healthcare providers.

Because masks alone are not effective without combining infection-control measures, ${ }^{7}$ we recommend this integrative platform for the maintenance of more PPE stockpiles, including critical infection-control equipment to reduce iatrogenic SARS-CoV-2 exposure.

\section{Acknowledgments.}

Financial support. No financial support was provided relevant to this article. article.

\section{References}

1. Wang MW, Cheng YR, Ye L, Zhou MY. The COVID-19 outbreak: the issue of face masks. Infect Control Hosp Epidemiol 2020 April 14 [Epub ahead of print]. doi: 10.1017/ice.2020.129.

2. Vigilante K, Escaravage S, McConnell M. big data and the intelligence community-lessons for health care. N Engl J Med 2019;380:1888-1890.

3. Wang CJ, Ng CY, Brook RH. Response to COVID-19 in Taiwan: big data analytics, new technology, and proactive testing. JAMA 2020;323:1341-1342.

4. Ranney ML, Griffeth V, Jha AK. Critical supply shortages- the need for ventilators and personal protective equipment during the COVID-19 pandemic. N Engl J Med 2020;382:e41. doi: 10.1056/NEJMp2006141.

5. National Health Command Center. Taiwan Centers for Disease Control website. https://www.cdc.gov.tw/En/Category/MPage/gL7-bARtHyNdrDq882pJ9Q. Updated February 1, 2018. Accessed May 6, 2020.

6. Hale T, Webster S, Petherick A, Phillips T, Kira B. Oxford COVID-19 government response tracker. Blavatnik School of Government. Data use policy: Creative Commons Attribution CC BY standard. 2020.

7. Klompas M, Morris CA, Sinclair J, Pearson M, Shenoy ES. Universal masking in hospitals in the COVID-19 era. NEngl J Med 2020. doi: 10.1056/NEJMp2006372.

\title{
Impact of early carbapenemase notification on infection control management and antimicrobial stewardship
}

\author{
Leandro Reus Rodrigues Perez $\mathrm{PhD}^{1}$ (1), Gabriel Narvaez $\mathrm{MD}^{2}$ and Cícero Dias PhD \\ ${ }^{1}$ Federal University of Health Sciences of Porto Alegre, Porto Alegre, Brazil and ${ }^{2}$ Mãe de Deus Hospital, Porto Alegre, Brazil
}

To the Editor-The worldwide spreading of carbapenemaseproducing Enterobacterales (CPE) is a matter of concern due to the limited therapeutic options available. ${ }^{1}$ In severe cases of infection, an early carbapenemase detection and notification is crucially important for the adequacy of antimicrobial treatment, for the management of patients, and to establish infection control practices. $^{2}$ Some microbiology laboratories have used blue-carba, a colorimetric test, because it is fast, easy to read, and inexpensive. ${ }^{3}$ However, the impact for the infection control practices and the turnaround time of its use on previous carbapenemase detection among Enterobacterales has been poorly evaluated.

We aimed to determine the turnaround time until CPE notification in comparison with the time to report a final microbiology result (bacterial identification plus antimicrobial susceptibility testing). We also aimed to evaluate the importance of this notification for the infection control measures and antimicrobial resistance predictability.

During a follow-up survey from August 2017 to August 2018, we performed an observational study in patients at a tertiary-care hospital from Porto Alegre, Brazil. Enterobacterales isolates

Author for correspondence: Leandro Reus Rodrigues Perez, E-mail: leandro.reus@ gmail.com

PREVIOUS PRESENTATION. This information was presented in part as abstract no. ECCMD-5455 in the "Better together: antibiotic stewardship and infection control" session of the $30^{\text {th }}$ European Congress of Clinical Microbiology and Infectious Diseases in Paris, France, on April 21, 2020.

Cite this article: Perez LRR, Narvaez G, and Dias C. (2022). Impact of early carbapenemase notification on infection control management and antimicrobial stewardship. Infection Control \& Hospital Epidemiology, 43: 134-136, https://doi.org/ 10.1017/ice.2020.1391 recovered from any clinical specimen were submitted to blue-carba test (BCT) for phenotypic carbapenemase detection. ${ }^{3}$ Isolates were identified using Vitek 2 (bioMérieux, Marcy l'Etoile, France) or MALDI-TOF/MS (Bruker Daltonics, Germany), if necessary. Antimicrobial susceptibility testing was determined by disc diffusion (Oxoid, for amikacin, gentamycin, meropenem; Etest (bioMérieux, Marc l'Étoile, France) for fosfomycin (when isolates were recovered from urine) and broth microdilution for polymyxin $\mathrm{B}$ and tigecycline. Carbapenemase characterization was conducted using phenotypic tests using specific inhibitors, as described elsewhere. 4

The work flow required the microbiology laboratory to notify the infection control staff or clinician of a positive BCT result for early carbapenemase notification after bacterial isolation from each clinical specimen analyzed.

During the period of the study, 300 CPE notifications were made, including 155 distinct patients. The average time was 1.19 days for CPE notification versus 2.38 days for the final report (Fig. 1). KPC-producing Klebsiella pneumoniae was the most prevalent agent (291 of $300,97 \%$ ) and no other gene carbapenemase than $b l a_{\mathrm{KPC}-2}$ was detected during this period. Antimicrobial resistance was observed as follows: meropenem 97.7\%, gentamicin 77.6\%, fosfomycin 31.6\%, polymyxin B $29.0 \%$, amikacin $7.3 \%$ and tigecycline $5 \%$.

Of the 155 patients enrolled in this survey, in 73 patients (47.1\%) an adjustment of antimicrobial therapy was promoted after the early BCT notification. These adjustments were due to the inclusion of polymyxin B $(65.7 \%, 48$ patients), amikacin (28.8\%, 21 patients), or fosfomycin (5.5\%, 4 patients). For 25 patients $(16.1 \%)$, no change in initial therapy was verified. In these 


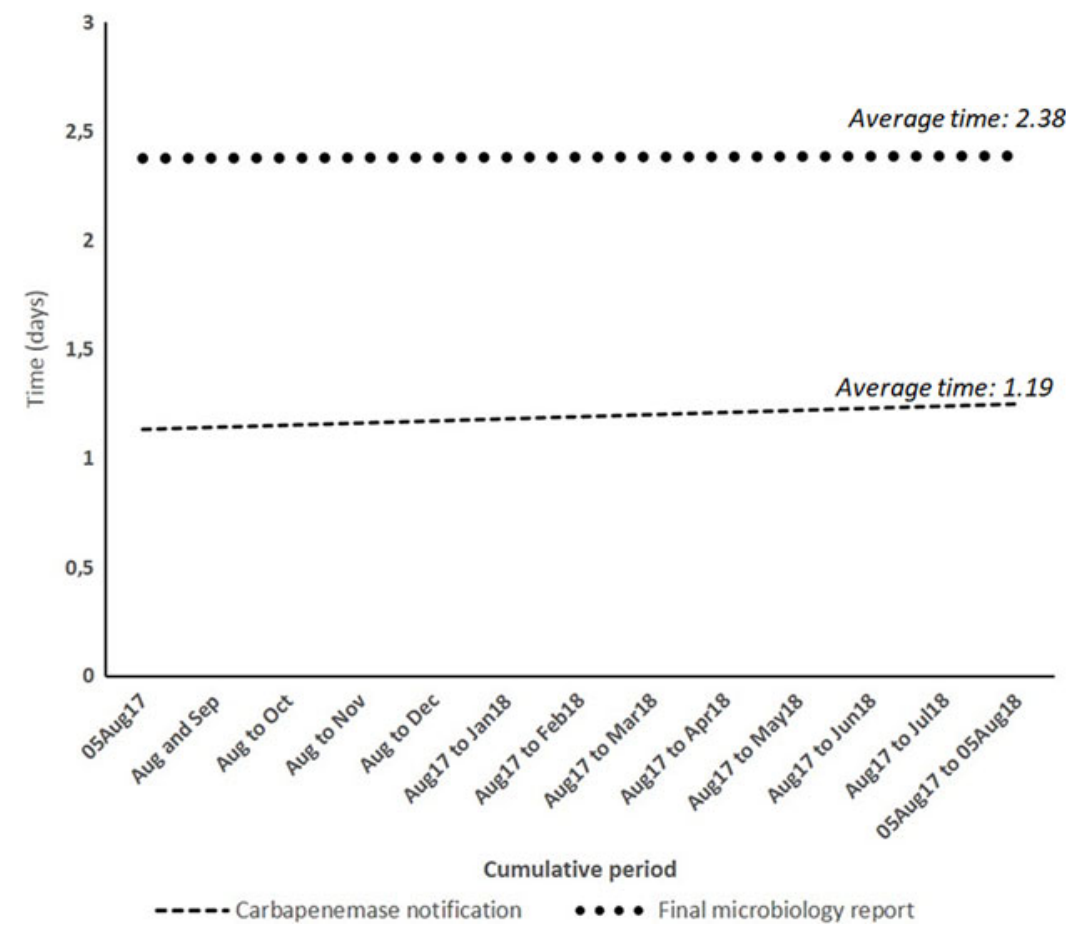

Fig. 1. Average times from an early carbapenemase notification by blue-carba test and from a final microbiology report during the study period.

cases, an inadequate therapy was considered because the antimicrobial agent administered did not present an in vitro susceptibility or was not directed toward the presence of a carbapenemase-producing organism even without susceptibility later detected. Also, 48 patients $(31.0 \%)$ were considered be colonized by CPE and for these, the therapeutic approaches after BCT notification was not evaluated. The remaining 9 patients $(5.8 \%)$ were from ambulatory origin, were discharged or died and, therefore, it was not possible to assess the therapeutic follow-up and impact of early carbapenemase notification.

Considering that early appropriate antimicrobial therapy can be the most important modifiable factor able to gain better patient's outcomes, BCT results may play a crucial role in decision making regarding therapy in infections in which CPEs occurred., Concordantly, we have shown the importance of an early BCT result when applied in infections caused by Pseudomonas aeruginosa isolates.?

For the infection control point of view, in our study, for 25 patients (16\%) and 121 patients (78\%) anticipated the installation of standard and contact precaution, respectively, based on an early BCT notification. On the other hand, from a clinical point of view, this fact means that an active antimicrobial agent (mostly polymyxin B in our study) should be initiated or included for adequacy of therapy.

The potential limitations of this study are related to the lack of control over variables related to the illness and patient outcome to determine the efficacy of an earlier intervention on the initial inadequacy of antimicrobial chemotherapy. Importantly, a BCT negative result may provide a better turnaround time for de-escalation practice, with a more strict and selective use of key antimicrobial agents, such as polymyxin B or ceftazidime/avibactam, according to the best practices of antimicrobial stewardship. ${ }^{8}$

In conclusion, CPE notification allows a shorter turnaround time for an earlier intervention (at least 24 hours, see Fig. 1) when compared with the final report. For $47.1 \%$ of patients, an early adjustment of therapy was done according to knowledge of the local epidemiological profile, particularly by use of an antimicrobial agent with in vitro activity. Active communication between laboratory and clinical services is mandatory to better explore this notification, significantly reducing the time to a first intervention.

Acknowledgment. We thank the staff of the infection control service of the Mãe de Deus Hospital for their support.

Financial support. No financial support was provided relevant to this article.

Conflicts of interest. All authors report no conflicts of interest relevant to this article.

\section{References}

1. Nordmann, P, Naas, T, Poirel, L. Global spread of carbapenemase-producing Enterobacteriaceae. Emerg Infect Dis 2011;17:1791-1798.

2. Rodrigues Perez LR, Dias CG. Emergence of infections due to a polymyxin B-resistant KPC-2-producing Klebsiella pneumoniae in critically ill patients: what is the role of a previous colonization? Infect Control Hosp Epidemiol 2016;37:240-241.

3. Pires J, Novais A, Peixe L. Blue-Carba, an easy biochemical test for detection of diverse carbapenemase producers directly from bacterial cultures. J Clin Microbiol 2013;51:4281-4283. 
4. Perez, LR. Carbapenem-Resistant Enterobacteriaceae: a major prevalence difference due to the high performance of carbapenemase producers when compared to the nonproducers. Infect Control Hosp Epidemiol 2015;36:1480-1482.

5. Tamma PD, Goodman KE, Harris AD, et al. Comparing the outcomes of patients with carbapenemase-producing and non-carbapenemase-producing carbapenem-resistant Enterobacteriaceae bacteremia. Clin Infect Dis 2017;64:257-264.

6. Falcone M, Bassetti M, Tiseo G, et al. Time to appropriate antibiotic therapy is a predictor of outcome in patients with bloodstream infection caused by KPC-producing Klebsiella pneumoniae. Crit Care 2020;24:29.
7. da Cunha RSR, Carniel E, Narvaez GA, Dias CG, Perez LRR. Impact of the blue-carba rapid test for carbapenemase detection on turnaround time for an early therapy against Pseudomonas aeruginosa. Am J Infect Control 2020. doi: 10.1016/j.ajic.2020.08.018.

8. Trivedi K, Dumartin C, Gilchrist M, Wade P, Howard P. Identifying best practices across three countries: hospital antimicrobial stewardship in the United Kingdom, France, and the United States. Clin Infect Dis 2014;59: S170-S178. 